\title{
The extremal graphs with respect to their nullity
}

Sa Rula ${ }^{1 *}$, An Chang ${ }^{1}$ and Yirong Zheng ${ }^{1,2}$

\section{"Correspondence:}

changsarula163@163.com

'Center for Discrete Mathematics,

Fuzhou University, Fuzhou, Fujian,

P.R. China

Full list of author information is

available at the end of the article
MSC: 05C50

Keywords: nullity of graph; matching number

\section{Introduction}

All graphs considered in this paper are finite, undirected, and simple. Let $G$ be a graph of order $n(G)$ and size $e(G)$. The adjacency matrix of $G$, denoted by $A(G)=\left(a_{i j}\right)_{n \times n}$, is the $n \times n$ matrix such that $a_{i j}=1$ if vertices $v_{i}$ and $v_{j}$ are adjacent and $a_{i j}=0$ otherwise, $i, j=$ $1, \ldots, n$. The rank of a graph $G$, denoted by $r(G)$, is the rank of its adjacency matrix $A(G)$. The multiplicity of the eigenvalue zero of $A(G)$ is called the nullity of $G$ and is denoted by $\eta(G)$. It is obvious that $\eta(G)=n(G)-r(G)$. The graph $G$ is called singular if $\eta(G)>0$ and called nonsingular if $\eta(G)=0$. Collatz and Sinogowitz [1] first raised the problem of characterizing all singular or nonsingular graphs. This problem is hard to be solved. The nullity is relevant to the rank of symmetric matrices described by graphs. On the other hand, the nullity has strong chemical background. A singular bipartite graph expresses the chemical instability of the molecule corresponding to the bipartite graph. Due to all these reasons, the nullity aroused the interest of many mathematicians and chemists. The topics on the nullity of graphs includes the computing nullity, the nullity distribution, bounds on nullity, characterization of graphs with certain nullity, and so on. For some particular classes of graphs, some preliminary results about nullity have been released [2-14].

It is known that $0 \leq \eta(G) \leq n(G)-2$ if $G$ is a graph of order $n(G)$ containing at least one edge [15]. Cheng and Liu [2] characterized all graphs with nullity $n(G)-2$ and $n(G)-3$. Chang et al. $[3,4]$ characterized all graphs of ranks 4 and 5 (i.e., with nullity $n(G)-4$ and $n(G)-5)$. Along this way, Zhu et al. [14] gave the characterization of the unicyclic graphs with nullity $n(G)-6$ and $n(G)-7$. Li et al. [10] characterized two kinds of bicyclic graphs with $n(G)-6$, whereas the entire characterization of graphs with nullity $n(G)-6, n(G)-7$, etc. is still unfinished. 
A matching in a graph $G$ is a set of pairwise nonadjacent edges. A maximum matching is one that contains as many edges of $G$ as possible. The matching number of $G$, denoted by $m(G)$, is the size of a maximum matching in $G$. Let $c(G)=e(G)-n(G)+\omega(G)$, where $\omega(G)$ is the number of connected components of $G$. Wang and Wong [16] obtained the following bounds of the matching number.

Theorem 1.1 ([16]) Let $G$ be a graph of rank $r(G)$. Then

$$
\left\lceil\frac{r(G)-c(G)}{2}\right\rceil \leq m(G) \leq\left\lfloor\frac{r(G)+2 c(G)}{2}\right\rfloor
$$

Since $\eta(G)=n(G)-r(G)$, this theorem can be equivalently rewritten in the following form in terms of the nullity of $G$.

Theorem 1.2 Let $G$ be a graph with nullity $\eta(G)$. Then

$$
n(G)-2 m(G)-c(G) \leq \eta(G) \leq n(G)-2 m(G)+2 c(G)
$$

Recently, Song et al. [12] characterized all graphs attaining the upper bound, that is, the graphs with nullity $\eta(G)=n(G)-2 m(G)+2 c(G)$.

Theorem 1.3 ([12]) For any graph $G, \eta(G)=n(G)-2 m(G)+2 c(G)$ if and only if the following three conditions are all satisfied:

(1) All cycles of $G$ are pairwise vertex-disjoint.

(2) The length of each cycle of $G$ is a multiple of 4.

(3) $m\left(T_{G}\right)=m\left(T_{G}-W_{G}\right)$, where $T_{G}$ is obtained from $G$ by contracting each cycle into a vertex, and $W_{G}$ consists of new vertices obtained by contracting each cycle of $G$.

Motivated by the works mentioned, in this paper, we characterize all graphs $G$ with nullity $\eta(G)=n(G)-2 m(G)-c(G)$. The main result is as follows.

Theorem 1.4 For any graph $G, \eta(G)=n(G)-2 m(G)-c(G)$ if and only if the following three conditions are all satisfied:

(a) Cycles of $G$ (if any) are pairwise vertex-disjoint.

(b) Each cycle of $G$ (if any) is odd cycle.

(c) $m\left(T_{G}\right)=m\left(T_{G}-W_{G}\right)$.

\section{Preliminaries}

Let $G$ be a graph with vertex set $V(G)$. We denote by $G-U$ the graph obtained from $G$ by removing the vertices in $U$ together with all incident edges, where $U \subseteq V(G)$. Especially, when $U=\{x\}$, we write $G-U$ simply as $G-x$. Sometimes we use the notation $G-G_{1}$ instead of $G-V\left(G_{1}\right)$ when $G_{1}$ is an induced subgraph of $G$. If $G_{1}$ is an induced subgraph of $G$ and $x$ is a vertex not in $G_{1}$, we write the subgraph of $G$ induced by $V\left(G_{1}\right) \cup\{x\}$ simply as $G_{1}+x$.

Let $C_{n}$ and $P_{n}$ be the cycle and path on $n$ vertices, respectively. Let $B(l, x, k)$ be the graph shown in Figure 1, which is obtained from two vertex-disjoint cycles $C_{l}$ and $C_{k}$ by connecting $v_{1} \in V\left(C_{l}\right)$ and $v_{x} \in V\left(C_{k}\right)$ with a path $P_{x}$ of length $x$, where $l, k \geq 3$ and $x \geq 1$ (when 
Figure $1 B(I, x, k)$ and $\theta(l, x, k)$.

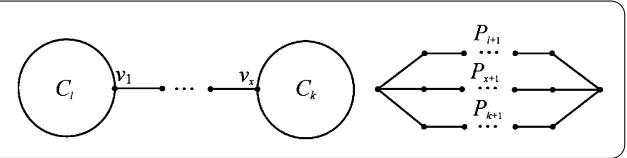

$x=1$, it means that we identify $v_{1}$ and $\left.v_{x}\right)$. Let $\theta(l, x, k)$ be the graph shown in Figure 1 , which is the union of three internally disjoint paths $P_{l+1}, P_{x+1}$, and $P_{k+1}$ of lengths $l, x, k$, respectively, with common end vertices, where $l, x, k \geq 1$, and at most one of them is 1 .

The degree of a vertex $v$ in $G$, denoted by $d_{G}(v)$, is the number of edges incident to the vertex. A vertex is called a pendant vertex if its degree is 1 . A lollipop is a graph obtained by identifying a vertex on a cycle and an end vertex on a path. The following is a list of known results about nullity that we will need in the proofs.

Lemma 2.1 ([17]) Let $G=G_{1} \cup G_{2} \cup \cdots \cup G_{\omega}$, where $G_{1}, G_{2}, \ldots, G_{\omega}$ are connected components of $G$. Then

$$
\eta(G)=\sum_{i=1}^{\omega} \eta\left(G_{i}\right)
$$

Lemma 2.2 ([17]) Let $G$ be a graph containing a pendant vertex, and let $H$ be the induced subgraph of $G$ obtained by deleting the pendant vertex together with the vertex adjacent to it. Then $\eta(G)=\eta(H)$.

Lemma $2.3([15])$

$$
\eta\left(P_{n}\right)=\left\{\begin{array}{ll}
1, & n \text { is odd }, \\
0, & n \text { is even; }
\end{array} \quad \eta\left(C_{n}\right)= \begin{cases}2, & n \text { is a multiple of } 4 \\
0 & \text { otherwise }\end{cases}\right.
$$

Lemma 2.4 ([15]) For any vertex $x$ of graph $G, \eta(G)-1 \leq \eta(G-x) \leq \eta(G)+1$.

Lemma 2.5 ([7]) Let $x$ be a cut-vertex of a graph $G$, and $G_{1}$ be a component of $G-x$. If $\eta\left(G_{1}\right)=\eta\left(G_{1}+x\right)-1$, then $\eta(G)=\eta\left(G_{1}\right)+\eta\left(G-G_{1}\right)$.

Lemma 2.6 ([12]) Let $G$ be a graph with at least one cycle. Then the cycles of $G$ are pairwise vertex-disjoint if and only iffor any vertex $u$ of $G$ that lies on a cycle, $c(G-u)=c(G)-1$.

Lemma 2.7 ([12]) Let $u$ be a pendant vertex of a graph $G$, and $v$ be the vertex adjacent to $u$. Denote $G_{0}=G-\{u, v\}$. Then, $m\left(T_{G}\right)=m\left(T_{G}-W_{G}\right)$ if and only if $v$ does not lie on a cycle and $m\left(T_{G_{0}}\right)=m\left(T_{G_{0}}-W_{G_{0}}\right)$.

Lemma 2.8 ([15]) For every acyclic graph $F$ with at least one vertex,

$$
\eta(F)=n(F)-2 m(F)
$$

For convenience, hereafter in this paper, we say a graph $G$ satisfies the minimal nullity condition if $\eta(G)=n(G)-2 m(G)-c(G)$. For acyclic graph $F$, since $c(F)=0$, it follows from Lemma 2.8 that every acyclic graph satisfies the minimal nullity condition. For a connected graph $G$, when $c(G)=1, G$ must be a unicyclic graph (a connected graph with 
equal number of vertices and edges). Guo et al. [8] characterized all unicyclic graphs that satisfy the minimal nullity condition.

Lemma 2.9 ([8]) Let $G$ be a unicyclic graph, and let $C_{l}$ be the unique cycle of $G$ of length $l$. Then $\eta(G)=n(G)-2 m(G)-1$ if and only if $l$ is odd and $m(G)=\frac{l-1}{2}+m\left(G-C_{l}\right)$.

The next lemma follows immediately from Lemma 2.9.

Lemma 2.10 Among all cycles, only odd cycles satisfy the minimal nullity condition.

Lemma 2.11 Let $u$ be a pendant vertex of a graph $G$ and $v$ be the vertex adjacent to $u$. Denote $G-\{u, v\}$ by $G_{0}$. Then, $G$ satisfies the minimal nullity condition if and only if $v$ does not lie on a cycle of $G$ and $G_{0}$ satisfies the minimal nullity condition.

Proof By Lemma 2.2, we know that $\eta\left(G_{0}\right)=\eta(G)$. Clearly, $m\left(G_{0}\right)=m(G)-1$.

' $\Leftarrow$ ' Since $v$ does not lie on a cycle of $G$, we have $c\left(G_{0}\right)=c(G)$. Moreover, $G_{0}$ satisfies the minimal nullity condition, then we have

$$
\begin{aligned}
\eta(G) & =\eta\left(G_{0}\right)=n\left(G_{0}\right)-2 m\left(G_{0}\right)-c\left(G_{0}\right) \\
& =(n(G)-2)-2(m(G)-1)-c(G) \\
& =n(G)-2 m(G)-c(G),
\end{aligned}
$$

which means that $G$ satisfies the minimal nullity condition.

' $\Rightarrow$ ' If $v$ lies on a cycle, then we have $c\left(G_{0}\right) \leq c(G)-1$. Since $G$ satisfies the minimal nullity condition, we have

$$
\begin{aligned}
\eta\left(G_{0}\right) & =\eta(G)=n(G)-2 m(G)-c(G) \\
& \leq\left(n\left(G_{0}\right)+2\right)-2\left(m\left(G_{0}\right)+1\right)-\left(c\left(G_{0}\right)+1\right) \\
& =n\left(G_{0}\right)-2 m\left(G_{0}\right)-c\left(G_{0}\right)-1,
\end{aligned}
$$

which is contradict with $\eta\left(G_{0}\right) \geq n\left(G_{0}\right)-2 m\left(G_{0}\right)-c\left(G_{0}\right)$. Therefore, $v$ does not lie on any cycle of $G$, then $c\left(G_{0}\right)=c(G)$. We have

$$
\begin{aligned}
\eta\left(G_{0}\right) & =\eta(G)=n(G)-2 m(G)-c(G) \\
& =\left(n\left(G_{0}\right)+2\right)-2\left(m\left(G_{0}\right)+1\right)-c\left(G_{0}\right) \\
& =n\left(G_{0}\right)-2 m\left(G_{0}\right)-c\left(G_{0}\right) \leq \eta\left(G_{0}\right),
\end{aligned}
$$

which means that $G_{0}$ satisfies the minimal nullity condition.

Lemma 2.12 Let $G$ be a graph with at least one cycle. Suppose that all cycles of $G$ are pairwise vertex-disjoint and each cycle of $G$ is odd. Then $m\left(T_{G}\right)=m\left(T_{G}-W_{G}\right)$ if and only if $m(G)=\sum_{C \in G} m(C)+m\left(G-\bigcup_{C \in G} C\right)$.

Proof Let $C_{1}, \ldots, C_{c(G)}$ be all cycles of $G$. Suppose that $G-\bigcup_{i=1}^{c(G)} C_{i}$ has $q$ connected components, say $H_{1}, \ldots, H_{q}$. Then $H_{1}, \ldots, H_{q}$ are also all components of $T_{G}-W_{G}$ since $T_{G}-W_{G}=G-\bigcup_{i=1}^{c(G)} C_{i}$. 
For $k=1, \ldots, q$, we can obtain the subgraph $\tilde{H}_{k}$ in $T_{G}$ induced by $V\left(H_{k}\right)$ and its all neighbor vertices. In $G$, we also can obtain the subgraph $\hat{H}_{k}$ induced by $V\left(H_{k}\right)$ and its all neighbor vertices. There is at most one edge between $V\left(H_{k}\right)$ and $V\left(C_{i}\right)$ since $H_{k}$ is connected and the cycles of $G$ are pairwise vertex-disjoint. Therefore, for each $k$, the graphs $\tilde{H}_{k}$ and $\hat{H}_{k}$ are isomorphic.

' $\Rightarrow$ ' Since $m\left(T_{G}\right)=m\left(T_{G}-W_{G}\right)$ and $m\left(T_{G}-W_{G}\right)=\sum_{k=1}^{q} m\left(H_{k}\right)$, we have $m\left(T_{G}\right)=$ $\sum_{k=1}^{q} m\left(H_{k}\right)$. Let $M_{T}$ be a maximum matching of $T_{G}$. Then $\left|M_{T}\right|=\sum_{k=1}^{q} m\left(H_{k}\right)$, which implies that $m\left(H_{k}\right)=m\left(\tilde{H}_{k}\right)$ for $k=1, \ldots, q$. Otherwise, there is $k_{0} \in\{1, \ldots, q\}$ such that $m\left(H_{k_{0}}\right)<m\left(\tilde{H}_{k_{0}}\right)$. Then we can easily get the matching $M_{T}^{\prime}$ of $T_{G}$ obtained from $M_{T}$ by replacing the maximum matching of $H_{k_{0}}$ by the maximum matching of $\tilde{H}_{k_{0}}$. Then we have

$$
\begin{aligned}
\left|M_{T}^{\prime}\right| & =m\left(\tilde{H}_{k_{0}}\right)+\sum_{k \neq k_{0}} m\left(H_{k}\right) \\
& >m\left(H_{k_{0}}\right)+\sum_{k \neq k_{0}} m\left(H_{k}\right) \\
& =\left|M_{T}\right|,
\end{aligned}
$$

which leads to a contradiction. Thus, $m\left(H_{k}\right)=m\left(\hat{H}_{k}\right), k=1, \ldots, q$, since $\tilde{H}_{k}$ and $\hat{H}_{k}$ are isomorphic.

Let $M$ be a maximum matching of $G$. Then we have

$$
\begin{aligned}
|M| & =\sum_{i=1}^{c(G)}\left|M \cap E\left(C_{i}\right)\right|+\sum_{k=1}^{q}\left|M \cap E\left(\hat{H}_{k}\right)\right| \\
& \leq \sum_{i=1}^{c(G)} m\left(C_{i}\right)+\sum_{k=1}^{q} m\left(\hat{H}_{k}\right) .
\end{aligned}
$$

On the other hand, $|M|=m(G) \geq \sum_{i=1}^{c(G)} m\left(C_{i}\right)+\sum_{k=1}^{q} m\left(H_{k}\right)$. Since $\sum_{k=1}^{q} m\left(H_{k}\right)=$ $\sum_{k=1}^{q} m\left(\hat{H}_{k}\right)$, we then have

$$
\begin{aligned}
|M| & =\sum_{i=1}^{c(G)} m\left(C_{i}\right)+\sum_{k=1}^{q} m\left(H_{k}\right) \\
& =\sum_{i=1}^{c(G)} m\left(C_{i}\right)+m\left(G-\bigcup_{i=1}^{c(G)} C_{i}\right) .
\end{aligned}
$$

' $\Leftarrow$ ' From $m(G)=\sum_{C \in G} m(C)+m\left(G-\bigcup_{C \in G} C\right)$ we have $m(G)=\sum_{i=1}^{c(G)} m\left(C_{i}\right)+$ $\sum_{k=1}^{q} m\left(H_{k}\right)$. Let $M$ be a maximum matching of $G$. Then $|M|=\sum_{i=1}^{c(G)} m\left(C_{i}\right)+\sum_{k=1}^{q} m\left(H_{k}\right)$. Assume to the contrary that $m\left(T_{G}\right) \neq m\left(T_{G}-W_{G}\right)$. Then $m\left(T_{G}\right)>m\left(T_{G}-W_{G}\right)$. Since $m\left(T_{G}-W_{G}\right)=\sum_{k=1}^{q} m\left(H_{k}\right)$, we have $m\left(T_{G}\right)>\sum_{k=1}^{q} m\left(H_{k}\right)$. It means that there is $k_{0} \in$ $\{1, \ldots, q\}$ such that $m\left(\tilde{H}_{k_{0}}\right)>m\left(H_{k_{0}}\right)$. Since $\tilde{H}_{k_{0}}$ and $\hat{H}_{k_{0}}$ are isomorphic, we have $m\left(\hat{H}_{k_{0}}\right)=$ $m\left(\tilde{H}_{k_{0}}\right)>m\left(H_{k_{0}}\right)$.

Since there is at most one edge between $V\left(H_{k_{0}}\right)$ and $V\left(C_{i}\right)(i=1, \ldots, c(G))$, without loss of generality, we can assume that for $i=1, \ldots, x$, there is exactly one edge between $V\left(H_{k_{0}}\right)$ and $V\left(C_{i}\right)$. Let $v_{i} \in V\left(C_{i}\right)(i=1, \ldots, x)$ be the unique vertex adjacent to $H_{k_{0}}$. Then we have the matching $M^{\prime}$ of $G$ which obtained from $M$ by replacing the maximum matching of $H_{k_{0}}$ 
by the maximum matching of $\hat{H}_{k_{0}}$ and by adjusting the maximum matching of each $C_{i}$ to the one without covering the vertex $v_{i}, i=1, \ldots, x$. Then,

$$
\begin{aligned}
\left|M^{\prime}\right| & =m\left(\hat{H}_{k_{0}}\right)+\sum_{k \neq k_{0}} m\left(H_{k}\right)+\sum_{i=1}^{x} m\left(C_{i}\right)+\sum_{i=x+1}^{c(G)} m\left(C_{i}\right) \\
& >m\left(H_{k_{0}}\right)+\sum_{k \neq k_{0}} m\left(H_{k}\right)+\sum_{i=1}^{c(G)} m\left(C_{i}\right) \quad\left(\because \text { each } C_{i} \text { is an odd cycle }\right) \\
& =\sum_{k=1}^{q} m\left(H_{k}\right)+\sum_{i=1}^{c(G)} m\left(C_{i}\right) \\
& =|M|,
\end{aligned}
$$

which leads to a contradiction.

\section{Main results}

Proposition 3.1 Let $G$ be a connected graph without pendant vertices, and $c(G) \geq 2$. Then $G$ does not satisfy the minimal nullity condition.

Proof Since $c(G) \geq 2$, there are at least two different cycles in $G$. We distinguish the following two cases.

Case 1 . There exists a vertex $u$ on a cycle such that $c(G-u) \leq c(G)-3$.

If $G$ satisfies the minimal nullity condition, then we have

$$
\begin{aligned}
\eta(G) & =n(G)-2 m(G)-c(G) \\
& \leq[n(G-u)+1]-2 m(G-u)-[c(G-u)+3] \quad(\because m(G-u) \leq m(G)) \\
& \leq n(G-u)-2 m(G-u)-c(G-u)-2 \\
& \leq \eta(G-u)-2 \quad(\text { by Theorem 1.2). }
\end{aligned}
$$

By Lemma 2.4 we know that $\eta(G) \geq \eta(G-u)-1$, a contradiction.

Case 2. For any vertex $u$ on a cycle, $c(G-u) \geq c(G)-2$.

We first prove the following claim.

Claim Let $G$ be a connected graph without pendant vertices. Suppose that for any vertex $u$ on a cycle of $G, c(G-u) \geq c(G)-2(c(G) \geq 2)$. Then there are at most $c(G)-1$ vertices of $G$ not covered by its maximum matching.

In fact, if a vertex $u$ lies on a cycle of $G$, then $c(G-u) \leq c(G)-1$. We distinguish the following two cases.

Case a. For any vertex $u$ that lies on a cycle of $G, c(G-u)=c(G)-1$.

By Lemma 2.6 all cycles of $G$ are pairwise vertex-disjoint in this case. By induction on $c(G)$, when $c(G)=2$, $G$ becomes $B(l, x, k)(x \geq 2)$. Since $B(l, x, k)(x \geq 2)$ has a path as its spanning subgraph, there is at most $1=c(G)-1$ vertex not covered by its maximum matching. 
We assume that the claim holds for a connected graph $G$ without pendant vertices and $c(G)<l$ and all cycles of $G$ are pairwise vertex-disjoint. Then we consider the case $c(G)=l$ $(l>2)$.

Since all cycles of $G$ are pairwise vertex-disjoint, there must exist a subgraph $G_{1}$ that is a lollipop graph such that $G-G_{1}$ is a graph without pendant vertices. The cycles of $G-G_{1}$ are also pairwise vertex-disjoint since it is a subgraph of $G$. Clearly, $c\left(G-G_{1}\right)=c(G)-1=$ $l-1<l$. By the induction assumption there are at most $c\left(G-G_{1}\right)-1$ vertices of $G-G_{1}$ not covered by the maximum matching of $G-G_{1}$. It is easy to see that in the lollipop graph $G_{1}$, there is at most one vertex not covered by the maximum matching of $G_{1}$. Then, there are at most $c\left(G-G_{1}\right)-1+1=c(G)-1$ vertices of $G$ not covered by its maximum matching.

Case b. There is a vertex $u$ that lies on a cycle of $G$ such that $c(G-u)=c(G)-2$. Similarly to Case a, by induction on $c(G)$, when $c(G)=2$, $G$ becomes $B(l, 1, k)$ or $\theta(l, x, k)$. Since $B(l, 1, k)$ and $\theta(l, x, k)$ have a path as their spanning subgraph, there is at most $1=c(G)-1$ vertex not covered by their maximum matchings.

We assume that the claim holds for a connected graph $G$ without pendant vertices and $c(G)<l$ and there is a vertex $u$ that lies on a cycle of $G$ such that $c(G-u)=c(G)-2$. It is suffices to prove the claim in the case $c(G)=l(l>2)$.

Suppose that $G-u$ has $p$ connected components, say $H_{1}, H_{2}, \ldots, H_{p}$. Let $n\left(H_{i}\right)$ and $e\left(H_{i}\right)$ be the number of vertices and size of $H_{i}, i=1,2, \ldots, p$, respectively. Obviously,

$$
\begin{aligned}
& n(G)=\sum_{i=1}^{p} n\left(H_{i}\right)+1, \quad e(G)=\sum_{i=1}^{p} e\left(H_{i}\right)+d_{G}(u), \\
& c\left(H_{i}\right)=e\left(H_{i}\right)-n\left(H_{i}\right)+1, \quad c(G)=e(G)-n(G)+1, \\
& c(G-u)=\sum_{i=1}^{p} c\left(H_{i}\right) .
\end{aligned}
$$

For each $H_{i}$, we have $c\left(H_{i}\right)<c(G), i=1, \ldots, p$. Moreover, we have $d_{G}(u)=p+2$. In fact,

$$
\begin{aligned}
d_{G}(u) & =e(G)-\sum_{i=1}^{p} e\left(H_{i}\right) \\
& =(c(G)+n(G)-1)-\sum_{i=1}^{p} e\left(H_{i}\right) \\
& =(c(G-u)+2)+\left(\sum_{i=1}^{p} n\left(H_{i}\right)+1\right)-1-\sum_{i=1}^{p} e\left(H_{i}\right) \\
& =\left(\sum_{i=1}^{p} c\left(H_{i}\right)+2\right)+\sum_{i=1}^{p} n\left(H_{i}\right)-\sum_{i=1}^{p} e\left(H_{i}\right) \\
& =\sum_{i=1}^{p}\left(e\left(H_{i}\right)-n\left(H_{i}\right)+1\right)+2+\sum_{i=1}^{p} n\left(H_{i}\right)-\sum_{i=1}^{p} e\left(H_{i}\right) \\
& =\sum_{i=1}^{p} e\left(H_{i}\right)-\sum_{i=1}^{p} n\left(H_{i}\right)+p+2+\sum_{i=1}^{p} n\left(H_{i}\right)-\sum_{i=1}^{p} e\left(H_{i}\right) \\
& =p+2 .
\end{aligned}
$$




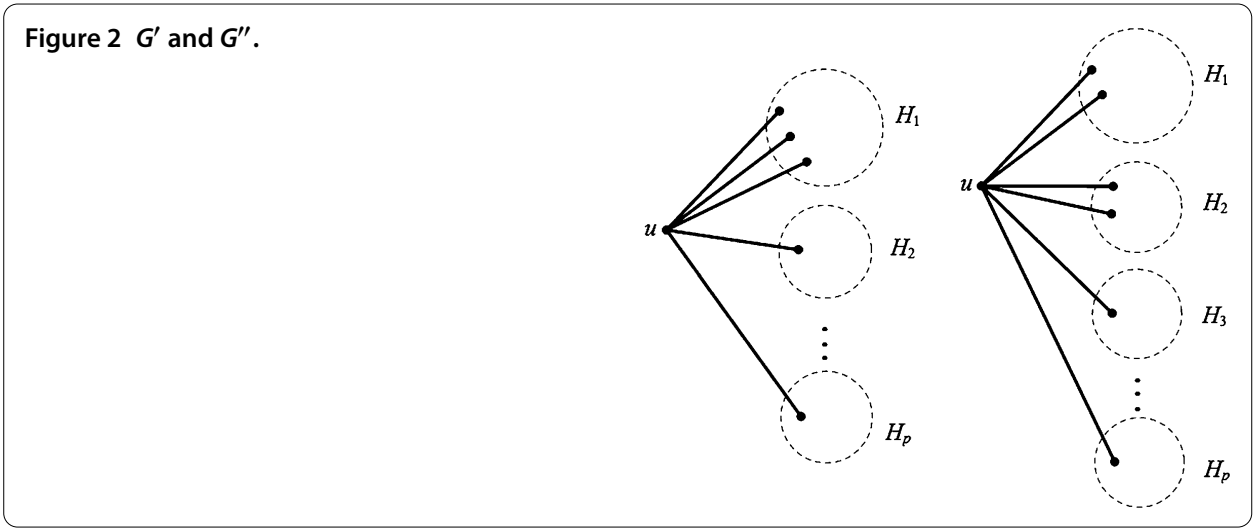

Thus, by the incident relation between $u$ and $H_{i}, G$ must be isomorphic to one of the two graphs shown in Figure 2.

Now, if $G$ is the graph $G^{\prime}$ shown in Figure 2, then we distinguish the following two cases.

Case b.1. Each component $H_{i}$ has no pendant vertices, $i=1,2, \ldots, p$.

Now assume that for $i=1, \ldots, s, c\left(H_{i}\right)=1$ and for $i=s+1, \ldots, p, c\left(H_{i}\right) \geq 2$. Then $c(G-u)=$ $\sum_{i=1}^{p} c\left(H_{i}\right)=s+c\left(H_{s+1}\right)+\cdots+c\left(H_{p}\right)$. When $i \in\{1, \ldots, s\}$, since $c\left(H_{i}\right)=1, H_{i}$ is a cycle and there is at most one vertex not covered by a maximum matching of $H_{i}$; when $i \in\{s+$ $1, \ldots, p\}$, since $c\left(H_{i}\right) \geq 2$, by the induction assumption there are at most $c\left(H_{i}\right)-1$ vertices not covered by a maximum matching of $H_{i}$. Let $V_{1}$ be a set of the vertices that are not be covered by a maximum matching of $G$. Then

$$
\begin{aligned}
\left|V_{1}\right| & \leq \underbrace{1+\cdots+1}_{s}+\left(c\left(H_{s+1}\right)-1\right)+\cdots+\left(c\left(H_{p}\right)-1\right)+|\{u\}| \\
& =s+c\left(H_{s+1}\right)+\cdots+c\left(H_{p}\right)-(p-s)+1 \\
& =c(G)-(p-s)-1 \quad\left(\because c(G)=c(G-u)+2=s+c\left(H_{s+1}\right)+\cdots+c\left(H_{p}\right)+2\right) \\
& \leq c(G)-1 \quad(\because p \geq s) .
\end{aligned}
$$

Thus, there are at most $c(G)-1$ vertices not overed by a maximum matching of $G$.

Case b.2. There exists a component, say $H_{i_{0}}\left(i_{0} \in\{1,2, \ldots, p\}\right)$ that has a pendant vertex. Let $v_{1}$ be the pendant vertex of $H_{i_{0}}$, and $P_{v_{1} v_{2} \cdots v_{x} v_{x+1}}$ be a path in $H_{i_{0}}$ such that $v_{1}$ is its end vertex and $d_{G}\left(v_{2}\right)=\cdots=d_{G}\left(v_{x}\right)=2, d_{G}\left(v_{x+1}\right)>2$. Clearly, $v_{1}$ is an adjacent vertex of $u$.

When $i_{0}=1$, by removing the path $P_{v_{1} v_{2} \cdots v_{x}}$ from $G$ the resulting graph $G-P_{v_{1} v_{2} \cdots v_{x}}$ is a graph without pendant vertex with $c\left(G-P_{v_{1} \nu_{2} \cdots \nu_{x}}\right)<c(G)$. By the induction assumption there are at most $c\left(G-P_{v_{1} v_{2} \cdots v_{x}}\right)-1$ vertices not covered by a maximum matching of $G-$ $P_{v_{1} v_{2} \cdots v_{x}}$. Recall that there is at most one vertex not covered by a maximum matching of the path $P_{v_{1} v_{2} \cdots v_{x}}$. Therefore, there are at most $c\left(G-P_{v_{1} v_{2} \cdots v_{x}}\right)-1+1 \leq c(G)-1$ vertices not covered by a maximum matching of $G$.

When $i_{0} \in\{2, \ldots, p\}$, by removing the path $P_{v_{1} v_{2} \cdots v_{x}}$ from $G$ the resulting graph $G-$ $P_{v_{1} v_{2} \cdots v_{x}}$ is a graph without pendant vertex, whereas $c\left(G-P_{v_{1} v_{2} \cdots v_{x}}\right)=c(G)$. It is obvious that $G-P_{\nu_{1} v_{2} \cdots v_{x}}$ has exactly two components, say $G_{1}$ and $G_{2}$, both without pendant vertices and with $c\left(G_{i}\right)<c(G), i=1,2$.

If $c\left(G_{i}\right) \geq 2, i=1,2$, by the induction assumption there are at most $c\left(G_{i}\right)-1$ vertices not covered by a maximum matching of $G_{i}$, respectively. Since there is at most one vertex not 
covered by a maximum matching of $P_{v_{1} v_{2} \cdots v_{x}}$, there are at most $c\left(G_{1}\right)-1+c\left(G_{2}\right)-1+1=$ $c(G)-1$ vertices not covered by a maximum matching of $G$.

If there is one of $G_{i}$, say $G_{1}$, with $c\left(G_{1}\right)=1$, then $P_{v_{1} v_{2} \cdots v_{x}}+G_{1}$ (the subgraph induced by $v_{1}, v_{2}, \ldots, v_{x}$ and $\left.V\left(G_{1}\right)\right)$ is a lollipop graph. Therefore, there is at most one vertex not covered by a maximum matching of $P_{v_{1} v_{2} \cdots v_{x}}+G_{1}$. Since $c(G) \geq 3$, we have $c\left(G_{2}\right) \geq 2$. By the induction assumption there are at most $c\left(G_{2}\right)-1$ vertices not covered by a maximum matching of $G_{2}$. Thus, there are at most $c\left(G_{2}\right)-1+1=c(G)-1$ vertices not covered by a maximum matching of $G$.

In a similar way, we can prove the claim when $G$ is the graph $G^{\prime \prime}$ shown in Figure 2.

By the previous claim, if $G$ satisfies the minimal nullity condition, then we have

$$
\begin{aligned}
\eta(G) & =n(G)-2 m(G)-c(G) \\
& \leq c(G)-1-c(G)=-1,
\end{aligned}
$$

a contradiction. This completes the proof of the lemma.

By Proposition 3.1 we have that if a connected graph $G$ with $c(G) \geq 2$ satisfies the minimal nullity condition, then $G$ must have a pendant vertex.

We are now ready to prove Theorem 1.4.

Proof It suffices to prove the theorem for the case where $G$ is connected.

' $\Rightarrow$ ' Let $G$ be a connected graph that satisfies the minimal nullity condition.

When $c(G)=0, G$ is a tree. Then (a), (b), and (c) hold trivially.

When $c(G)=1, G$ is a unicyclic graph. Let $C_{l}$ be the unique cycle of $G$ of length $l$. By Lemma 2.9, $C_{l}$ is an odd cycle, and $m(G)=\frac{l-1}{2}+m\left(G-C_{l}\right)$. Thus, (a) and (b) hold obviously. Moreover, since $m\left(C_{l}\right)=\frac{l-1}{2}$ and $m\left(G-C_{l}\right)=m\left(T_{G}-v_{C_{l}}\right)$, we have $m(G)=m\left(C_{l}\right)+m\left(T_{G}-\right.$ $\left.v_{C_{l}}\right)$. By Lemma 2.12, $m\left(T_{G}\right)=m\left(T_{G}-v_{C_{l}}\right)$. Thus, (c) holds.

It remains to prove (a), (b), and (c) for $G$ when $c(G) \geq 2$.

Assume that $G$ is a connected graph with $c(G) \geq 2$ that satisfies the minimal nullity condition. By Proposition 3.1, $G$ must have a pendant vertex. Let $u$ be a pendant vertex of G. It follows from Lemma 2.11 that the adjacent vertex $v$ of $u$ does not lie on a cycle of $G$, and by removing these two vertices $u$ and $v$ the resulting graph $G_{0}$ also satisfies the minimal nullity condition. Clearly, $c(G)=c\left(G_{0}\right) \geq 2$.

Let $H_{1}, H_{2}, \ldots, H_{p}$ be the connected components of $G_{0}$. Then each $H_{i}, i=1, \ldots, p$, satisfies the minimal nullity condition. Without loss of generality, we assume that for $i \in\{1, \ldots, s\}$, $c\left(H_{i}\right) \leq 1$ and for $i \in\{s+1, \ldots, p\}, c\left(H_{i}\right) \geq 2$. By Proposition 3.1, for $i \in\{s+1, \ldots, p\}$, each $H_{i}$ must have a pendant vertex, and by Lemma 2.11 the adjacent vertex of each pendant vertex does not lie on a cycle of $H_{i}$. By removing a pendant vertex of $H_{i}$ and its adjacent vertex the resulting graph also satisfies the minimal nullity condition. For a connected component with $c \geq 2$ that satisfies the minimal nullity condition, there must be a pendant vertex, and its adjacent vertex does not lie on a cycle. Then we can repeatedly remove a pendant vertex of $H_{i}, i \in\{s+1, \ldots, p\}$, and its adjacent vertex until all connected components of the resulting graph has $c \leq 1$. Continuing in a similar way, we can remove a pendant vertex and its adjacent vertex repeatedly until all connected components of the resulting graph do not contain pendant vertices. It is obvious that the final resulting graph, say $G^{*}$, consists only of cycles and isolated vertices. Furthermore, $G^{*}$ satisfies the minimal nullity condition 
and keeps $c\left(G^{*}\right)=c(G)$. It follows from Lemma 2.10 that $G^{*}$ is the disjoint union of $c(G)$ odd cycles, say $C_{1}, C_{2}, \ldots, C_{c(G)}$, and some isolated vertices. Therefore, the cycles of $G$ are pairwise vertex-disjoint, and each cycle is odd. Thus, (a) and (b) hold.

On the other hand, since in each step we just remove a pendant vertex and its adjacent vertex, we have $m(G)=\sum_{i=1}^{c(G)} m\left(C_{i}\right)+m\left(G-\bigcup_{i=1}^{c(G)} V\left(C_{i}\right)\right)$. Thus, by Lemma 2.12 we have $m\left(T_{G}\right)=m\left(T_{G}-W_{G}\right)$, and thus (c) holds.

' $\Leftarrow$ ' Let $G$ be a connected graph satisfying (a), (b), and (c). We deal with the sufficient part of the proof by induction on $c(G)$. When $c(G)=0, G$ is a tree. Then, by Lemma 2.8, the result is trivial. When $c(G)=1, G$ is a unicyclic graph. Let $C_{l}$ be the unique cycle of $G$ of length $l$. Then $C_{l}$ is an odd cycle, and $m\left(T_{G}\right)=m\left(T_{G}-v_{C_{l}}\right)$. By Lemma 2.12 we have $m(G)=m\left(C_{l}\right)+m\left(T_{G}-v_{C_{l}}\right)=\frac{l-1}{2}+m\left(G-C_{l}\right)$. Furthermore, by Lemma 2.9, $\eta(G)=n(G)-$ $2 m(G)-c(G)$ in this case.

We assume that the result holds when $c(G)<l$. Now we consider the case $c(G)=l(l \geq 2)$. Since cycles of $G$ are pairwise vertex-disjoint, there exists a cycle $C$ in $G$ such that all cycles of $G-C$ lie in the same component, say $H_{1}$, of $G-C$, and the other components of $G-C$, say $H_{i}(i=2, \ldots, p)$, are trees. Moreover, $n\left(H_{i}\right) \geq 2(i=2, \ldots, p)$. If not, there is $i_{0} \in\{2, \ldots, p\}$ such that $n\left(H_{i_{0}}\right)=1$, which means that there is a pendant vertex of $G$ such that its adjacent vertex lies on $C$. But this contradicts Lemma 2.7 because (c) holds for $G$ if and only if the adjacent vertex of any pendant vertex does not lie on a cycle.

If $p \geq 2$, then let $u$ be a pendant vertex of $G$ that belongs to $\bigcup_{i=2}^{p} V\left(H_{i}\right)$, and let $v$ be its adjacent vertex. Denote by $G_{0}$ the graph obtained from $G$ by removing $u$ and $v$. By Lemma 2.7, $v$ does not lie on a cycle of $G$, and (c) also holds for $G_{0}$. Repeating this operation, after a finite number of steps, we can obtain a graph $G^{*}$ that is a disjoint union of a connected component $G_{1}$ induced by $V(C) \cup V\left(H_{1}\right)$ and some acyclic graphs. Obviously, (c) also holds for $G^{*}$.

Since $G$ is a connected graph and all cycles are pairwise vertex-disjoint, there is exactly one edge between $C$ and $H_{1}$ in $G^{*}$. Let $x y$ is the unique edge between $C$ and $H_{1}$, where $x \in V(C)$ and $y \in V\left(H_{1}\right)$. Note that (c) holds for the graph $G^{*}$ if and only if (c) also holds for its each connected component. Therefore, (c) holds for $G_{1}$, that is, $m\left(T_{G_{1}}\right)=m\left(T_{G_{1}}-W_{G_{1}}\right)$. Certainly, (a) and (b) hold for $G_{1}$ since it is a subgraph of $G$. Moreover, both $H_{1}+x$ and $H_{1}$ also satisfy (a) and (b) since they are subgraphs of $G_{1}$. Now let us show that (c) also holds for $H_{1}+x$ and $H_{1}$, that is,

(1) $m\left(T_{H_{1}+x}\right)=m\left(T_{H_{1}+x}-W_{H_{1}+x}\right)$.

In fact, We have $m\left(T_{G_{1}}\right)=m\left(T_{H_{1}+x}\right)$ since $T_{G_{1}} \cong T_{H_{1}+x}$, and $W_{G_{1}}=W_{H_{1}+x} \cup\left\{v_{C}\right\}$, where $v_{C}$ is the vertex in $W_{G_{1}}$ contracted from the cycle $C$. Since $m\left(T_{G_{1}}\right)=m\left(T_{G_{1}}-W_{G_{1}}\right)$, we have

$$
\begin{aligned}
m\left(T_{H_{1}+x}\right) & =m\left(T_{H_{1}+x}-\left(W_{H_{1}+x} \cup\left\{v_{C}\right\}\right)\right) \\
& =m\left(T_{H_{1}+x}-W_{H_{1}+x}-v_{C}\right) \\
& \leq m\left(T_{H_{1}+x}-W_{H_{1}+x}\right) .
\end{aligned}
$$

On the other hand, it is clear that $m\left(T_{H_{1}+x}\right) \geq m\left(T_{H_{1}+x}-W_{H_{1}+x}\right)$. Therefore, $m\left(T_{H_{1}+x}\right)=$ $m\left(T_{H_{1}+x}-W_{H_{1}+x}\right)$.

(2) $m\left(T_{H_{1}}\right)=m\left(T_{H_{1}}-W_{H_{1}}\right)$. 
In fact, $T_{G_{1}}$ can be regarded as the graph induced by $V\left(T_{H_{1}}\right)$ and the vertex $v_{C}$. Therefore, $T_{G_{1}}=T_{H_{1}}+v_{C}$ and $W_{G_{1}}=W_{H_{1}} \cup\left\{v_{C}\right\}$. Since (c) holds for $G_{1}$, we have $m\left(T_{G_{1}}\right)=m\left(T_{G_{1}}-\right.$ $\left.W_{G_{1}}\right)$. Thus,

$$
\begin{aligned}
m\left(T_{H_{1}}+v_{C}\right) & =m\left(T_{H_{1}}+v_{C}-\left(W_{H_{1}} \cup\left\{v_{C}\right\}\right)\right) \\
& =m\left(T_{H_{1}}-W_{H_{1}}\right) \\
& \leq m\left(T_{H_{1}}\right) .
\end{aligned}
$$

Clearly, $m\left(T_{H_{1}}+v_{C}\right) \geq m\left(T_{H_{1}}\right)$. Then we have $m\left(T_{H_{1}}+v_{C}\right)=m\left(T_{H_{1}}\right)$. The previous derivation forces the last inequality involved to become an equality. Thus, $m\left(T_{H_{1}}\right)=m\left(T_{H_{1}}-\right.$ $\left.W_{H_{1}}\right)$.

Since $c\left(H_{1}+x\right)<c\left(G_{1}\right)=c(G)$ (respectively, $c\left(H_{1}\right)<c\left(G_{1}\right)=c(G)$ ), by the induction assumption, $H_{1}+x$ (respectively, $\left.H_{1}\right)$ satisfies the minimal nullity condition, that is, $\eta\left(H_{1}+\right.$ $x)=n\left(H_{1}+x\right)-2 m\left(H_{1}+x\right)-c\left(H_{1}+x\right)$ and $\eta\left(H_{1}\right)=n\left(H_{1}\right)-2 m\left(H_{1}\right)-c\left(H_{1}\right)$.

It follows from (1) and (2) that $m\left(T_{H_{1}+x}\right)=m\left(T_{G}\right)=m\left(T_{H_{1}}+v_{C}\right)=m\left(T_{H_{1}}\right)$, that is, $m\left(T_{H_{1}+x}\right)=m\left(T_{H_{1}}\right)$. Since $m\left(T_{H_{1}+x}\right)=m\left(T_{H_{1}+x}-W_{H_{1}+x}\right)$ and $m\left(T_{H_{1}}\right)=m\left(T_{H_{1}}-W_{H_{1}}\right)$, by Lemma 2.12 we have $m\left(H_{1}+x\right)=\sum_{C \in H_{1}+x} m(C)+m\left(T_{H_{1}+x}-W_{H_{1}+x}\right)$ and $m\left(H_{1}\right)=$ $\sum_{C \in H_{1}} m(C)+m\left(T_{H_{1}}-W_{H_{1}}\right)$. Then,

$$
\begin{aligned}
m\left(H_{1}+x\right) & =\sum_{C \in H_{1}+x} m(C)+m\left(T_{H_{1}+x}-W_{H_{1}+x}\right) \\
& =\sum_{C \in H_{1}+x} m(C)+m\left(T_{H_{1}+x}\right) \\
& =\sum_{C \in H_{1}} m(C)+m\left(T_{H_{1}}\right) \\
& =\sum_{C \in H_{1}} m(C)+m\left(T_{H_{1}}-W_{H_{1}}\right) \\
& =m\left(H_{1}\right)
\end{aligned}
$$

that is, $m\left(H_{1}+x\right)=m\left(H_{1}\right)$. Furthermore, since both $H_{1}+x$ and $H_{1}$ satisfy the minimal nullity condition, we have

$$
\begin{aligned}
\eta\left(H_{1}+x\right) & =n\left(H_{1}+x\right)-2 m\left(H_{1}+x\right)-c\left(H_{1}+x\right) \\
& =\left(n\left(H_{1}\right)+1\right)-2 m\left(H_{1}\right)-c\left(H_{1}\right) \\
& =\eta\left(H_{1}\right)+1 .
\end{aligned}
$$

Then by Lemma 2.5 we have

$$
\begin{aligned}
\eta\left(G_{1}\right) & =\eta\left(H_{1}\right)+\eta\left(G_{1}-H_{1}\right) \\
& =n\left(H_{1}\right)-2 m\left(H_{1}\right)-c\left(H_{1}\right)+\eta(C) \\
& =\left(n\left(G_{1}\right)-n(C)\right)-2(m(G)-m(C))-\left(c\left(G_{1}\right)-1\right)+0 \\
& =n\left(G_{1}\right)-2 m\left(G_{1}\right)-c\left(G_{1}\right),
\end{aligned}
$$


that is, $G_{1}$ satisfies the minimal nullity condition. On the other hand, each acyclic graph satisfies the minimal nullity condition. Then $G^{*}$ also satisfies the minimal nullity condition. It follows from Lemma 2.11 that $G$ satisfies the minimal nullity condition since $G^{*}$ does. The proof is completed.

Proposition 3.2 Let $G$ be a graph with at least one cycle. If $G$ satisfies the minimal nullity condition, then for any vertex $u$ that lies on a cycle of $G$, we have that $G-u$ also satisfies the minimal nullity condition and $\eta(G-u)=\eta(G)$.

Proof It suffices to prove the result for the case where $G$ is connected. Let $G$ be a connected graph satisfying the minimal nullity condition. By Theorem 1.4 all cycles of $G$, say $C_{1}, C_{2}, \ldots, C_{c(G)}$, are pairwise vertex-disjoint, and each $C_{i}(i=1, \ldots, c(G))$ is an odd cycle. Let $u$ be any vertex that lies on a cycle of $G$, say $C_{i_{0}}\left(i_{0} \in\{1, \ldots, c(G)\}\right)$. Since $G$ satisfies the minimal nullity condition, by Theorem 1.4 we have $m\left(T_{G}\right)=m\left(T_{G}-W_{G}\right)$. Then by Lemma 2.12 we have

$$
\begin{aligned}
m(G) & =\sum_{i=1}^{c(G)} m\left(C_{i}\right)+m\left(G-\bigcup_{i=1}^{c(G)} C_{i}\right) \\
& =\sum_{i \neq i_{0}} m\left(C_{i}\right)+m\left(C_{i_{0}}\right)+m\left((G-u)-\bigcup_{i \neq i_{0}} C_{i}-\left(C_{i_{0}}-u\right)\right) \\
& =\sum_{i \neq i_{0}} m\left(C_{i}\right)+m\left(C_{i_{0}}\right)+m\left((G-u)-\bigcup_{i \neq i_{0}} C_{i}\right)-m\left(C_{i_{0}}-u\right) \\
& =\sum_{i \neq i_{0}} m\left(C_{i}\right)+m\left((G-u)-\bigcup_{i \neq i_{0}} C_{i}\right) \\
& =\sum_{C_{i} \text { in } G-u} m\left(C_{i}\right)+m\left((G-u)-\bigcup_{C_{i} \text { in } G-u} C_{i}\right) \\
& \leq m(G-u) .
\end{aligned}
$$

However, $m(G) \geq m(G-u)$. Thus, we have $m(G)=m(G-u)$. Furthermore,

$$
m(G-u)=\sum_{C_{i} \text { in } G-u} m\left(C_{i}\right)+m\left((G-u)-\bigcup_{C_{i} \text { in } G-u} C_{i}\right) .
$$

By Lemma 2.12 we have $m\left(T_{G-u}\right)=m\left(T_{G-u}-W_{G-u}\right)$, that is, $G-u$ satisfies (c) of Theorem 1.4. Moreover, $G-u$ also satisfies both (a) and (b) of Theorem 1.4 since $G-u$ is a subgraph of $G$. So $G-u$ satisfies the minimal nullity condition. Then we have

$$
\begin{aligned}
\eta(G) & =n(G)-2 m(G)-c(G) \\
& =[n(G-u)+1]-2 m(G-u)-[c(G-u)+1] \quad(\because m(G-u)=m(G)) \\
& =n(G-u)-2 m(G-u)-c(G-u) \\
& =\eta(G-u)
\end{aligned}
$$

and the proof is completed. 


\section{Conclusions}

All graphs $G$ with $\eta(G)=n(G)-2 m(G)-c(G)$ are completely characterized. This means that we can easily obtain the nullity $\eta(G)$ of each of those graphs from its order $n(G)$, matching number $m(G)$, and elementary cyclic number $c(G)$, and, thus, we can know whether the graph is singular or not.

\section{Competing interests}

The authors declare that they have no competing interests.

\section{Authors' contributions}

SR carried out the proofs of the main results in the manuscript. AC and YZ participated in the design of the study and drafted the manuscripts. All the authors read and approved the final manuscripts.

\section{Author details}

${ }^{1}$ Center for Discrete Mathematics, Fuzhou University, Fuzhou, Fujian, P.R. China. ${ }^{2}$ School of Applied Mathematics, Xiamen University of Technology, Xiamen, Fujian, P.R. China.

\section{Acknowledgements}

The authors would like to thank the anonymous referees for their constructive corrections and valuable comments on this paper, which have considerably improved the presentation of this paper. This project is supported by NSF of China (Nos. 11331003, 11471077), China.

Received: 12 November 2015 Accepted: 3 February 2016 Published online: 20 February 2016

\section{References}

1. Collatz, L, Sinogowitz, U: Spektren endlicher Grafen. Abh. Math. Semin. Univ. Hamb. 21, 63-77 (1957)

2. Cheng, B, Liu, B: On the nullity of graphs. Electron. J. Linear Algebra 16, 60-67 (2007)

3. Chang, GJ, Huang, L-H, Yeh, H-G: A characterization of graphs with rank 4. Linear Algebra Appl. 434(8), 1793-1798 (2011)

4. Chang, GJ, Huang, L-H, Yeh, H-G: A characterization of graphs with rank 5. Linear Algebra Appl. 436(11), 4241-4250 (2012)

5. Fan, YZ, Qian, KS: On the nullity of bipartite graphs. Linear Algebra Appl. 430, 2943-2949 (2009)

6. Fiorini, S, Gutman, I, Sciriha, I: Trees with maximum nullity. Linear Algebra Appl. 397, 245-251 (2005)

7. Gong, SC, Xu, GH: On the nullity of a graph with cut-points. Linear Algebra Appl. 436, 135-142 (2012)

8. Guo, JM, Yan, WG, Yeh, YN: On the nullity and the matching number of unicyclic graphs. Linear Algebra Appl. 431, 1293-1301 (2009)

9. Hu, SB, Tan, XZ, Liu, BL: On the nullity of bicyclic graphs. Linear Algebra Appl. 429, 1387-1391 (2008)

10. Li, J, Chang, A, Shiu, WC: On the nullity of bicyclic graphs. MATCH Commun. Math. Comput. Chem. 60, 21-36 (2008)

11. Li, SC: On the nullity of graphs with pendant vertices. Linear Algebra Appl. 429, 1619-1628 (2008)

12. Song, $Y Z$, Song, $X Q$, Tam, BS: A characterization of graphs $G$ with nullity $|V(G)|-2 m(G)-c(G)$. Linear Algebra Appl. 465, 363-375 (2015)

13. Tan, X, Liu, B: On the nullity of unicyclic graphs. Linear Algebra Appl. 408, 212-220 (2005)

14. Zhu, W, Wu, TZ, Hu, SB: A note on the nullity of unicyclic graphs. J. Math. Res. Expo. 30(5), 817-824 (2010)

15. Brouwer, AE, Haemers, WH: Spectra of Graphs. Springer, New York (2011)

16. Wang, L, Wong, D: Bounds for the matching number, the edge chromatic number and the independence number of a graph in terms of rank. Discrete Appl. Math. 166, 276-281 (2014)

17. Cvetković, DM, Doob, M, Sachs, H: Spectra of Graphs: Theory and Application. Academic Press, New York (1980)

\section{Submit your manuscript to a SpringerOpen ${ }^{\ominus}$ journal and benefit from:}

- Convenient online submission

Rigorous peer review

- Immediate publication on acceptance

Open access: articles freely available online

- High visibility within the field

- Retaining the copyright to your article 